\title{
Application of rational Chebyshev polynomials to optical problems
}

$\begin{array}{ll}\text { I. N. Towers } & \text { Z. Jovanoski } \\ & \end{array}$

(Received 4 August 2008; revised 30 September 2008)

\begin{abstract}
We present the use of the rational Chebyshev polynomials for discretising the transverse dimension(s) of beam propagation problems within the field of nonlinear optics. How a beam propagates in an optical medium, whether linear or nonlinear, is a common problem and important in both theoretical studies and optical design. The infinite domain and convergence properties of these polynomials allows one to handle the boundary conditions with greater correctness than methods that impose periodic boundary conditions such as Fourier methods. The beam is propagated forward by exponential integration for fast and accurate numerical simulations. The techniques employed to solve the beam propagation problems are easily applied to problems in other fields with mathematically similar models.
\end{abstract}

See http://anziamj.austms.org.au/ojs/index.php/ANZIAMJ/article/view/ 1396 for this article, (C) Austral. Mathematical Soc. 2008. Published October 7, 2008. ISSN 1446-8735 


\section{Contents}

1 Introduction

C61

2 Rational Chebyshev polynomials

C63

3 Exponential integrators

C64

4 Results

C66

5 Conclusion

C70

References

C72

\section{Introduction}

Many problems in optics involve propagating a field forward in time/space and simulating how it behaves in a nonlinear medium. Such problems often have, at least in theory, boundary conditions at infinity. Traditionally, when solving the underlying partial differential equation (PDE) of the problem, one discretises the transverse dimension(s) with finite differences or Fourier modes and propagates the field using some kind of explicit or implicit time integration scheme. The domain of the problem is necessarily truncated to a suitably large size which allows the boundary conditions to be approximated.

The finite difference method is an easy to understand and easy to implement way of numerically handling derivatives in a PDE. The resulting system of linear equations involves a coefficient matrix which is typically sparse and banded. Fast algorithms exist for solving such linear systems of equations. However, to achieve high accuracy requires either a large number of points in the transverse dimension(s) or increasingly complex finite difference schemes which reduce the sparsity (and increase the number of bands) of the coeffi- 
cient matrix. A comparison of the accuracy between finite differences and spectral methods of differentiation is in the book by Trefethen [1].

Using Fourier modes to discretise the transverse derivatives is a form of spectral method. Therefore it has the advantage of high accuracy compared to finite difference methods for a comparable number of points. Further, the fact that differentiation becomes a simple matter of multiplication in Fourier transform space and that there exists a number of freely available software packages which perform the fast Fourier transform (FFT) [2], has made the Fourier method popular for solving propagation problems [3].

A consequence of using Fourier modes is that it imposes periodic boundary conditions on the problem. If the problem involves propagating a localised structure, which is typical of many optical problems, then the periodic boundary conditions effectively mean that an infinite chain of localised beams are being propagated. Unless the computational domain is taken to be sufficiently wide the tails of the beam 'feels' the presence of other pulses in the chain and this affects the dynamics of the beam. In addition, any part of the optical field which encounters one boundary, such as radiation shed from the beam, will re-enter the computation domain from the opposite side and interact with the beam. This is unphysical and to avoid it many schemes use absorbing boundary conditions or perfectly matched layers have been developed [4]. Although successful, such techniques do increase the complexity of any simulation code.

In order to maintain the accuracy of a spectral method but avoid the imposition of periodic boundary conditions we apply in this work the socalled rational Chebyshev polynomials (RCP) to the simulation of beams as governed by the $(1+1)$-dimensional nonlinear Schrödinger equation (NLS) and its extension, the $(1+1)$-dimensional and $(2+1)$-dimensional cubicquintic nonlinear Schrödinger equation (CQNLS). The RCP are transformed versions of the standard Chebyshev polynomials which are defined on the entire real line as opposed to $[-1,1]$. 


\section{Rational Chebyshev polynomials}

Chebyshev polynomials are a well known set of orthogonal polynomials and have proved useful for numerical analysis. They are a popular choice of basis set when employing spectral methods because Chebyshev polynomials can be thought of as a "Fourier cosine series in disguise" [5] and as such allow the use of existing FFT software.

The rational Chebyshev polynomials (RCP) are a rescaling of the standard Chebyshev polynomials so that their domain is the entire real line [6]. Following Boyd [6], we define $y \in(-\infty, \infty), x \in[-1,1]$, and $t \in[0, \pi]$ then the different domains are mapped onto one another through the relationships

$$
y=\frac{L x}{\sqrt{1-x^{2}}}=L \cot (t), \quad x=\cos (t),
$$

where $\mathrm{L}>0$ is a constant which is chosen arbitrarily. The $n$th rational Chebyshev is

$$
\operatorname{TB}_{\mathfrak{n}}(\mathrm{y})=\mathrm{T}_{\mathrm{n}}\left(\frac{\mathrm{y}}{\sqrt{\mathrm{L}^{2}+\mathrm{y}^{2}}}\right)=\cos \left[n \operatorname{arccot}\left(\frac{\mathrm{y}}{\mathrm{L}}\right)\right],
$$

where $T_{n}(x)$ is the standard Chebyshev polynomial.

Small values of $L$ cause the majority of the discretised points in the transverse dimension to be clumped near the origin. Increasing L 'pulls' the points out from the origin towards the boundary of the domain. The profile of the beam one is investigating and the expected position of any interesting dynamics guides one's choice for the value of $\mathrm{L}$. We typically use $\mathrm{L}$ in the range $4-20$.

One now generates the RCP from either the standard Chebyshev polynomials or from the Fourier series. Here we choose to work with Chebyshev polynomials rather than Fourier series. This choice is merely one of personal 
convenience but we expect, for problems where a large number of RCP need to be generated, that calculating those polynomials via a FFT routine will be quicker than using the recurrence relation that generates the standard Chebyshev polynomials.

The transformations of derivatives are readily calculated by application of the chain rule. We give only the first two derivatives but a comprehensive table is presented in Boyd's book [5]. If $u$ is a function given as a series of $T_{n}(x)$, then

$$
\frac{\partial u}{\partial y}=\frac{\left(1-x^{2}\right)^{3 / 2}}{L} \frac{\partial u}{\partial x}, \quad \frac{\partial^{2} u}{\partial y^{2}}=\frac{\left(1-x^{2}\right)^{2}}{L^{2}}\left\{\left(1-x^{2}\right) \frac{\partial^{2} u}{\partial x^{2}}-3 x \frac{\partial u}{\partial x}\right\} .
$$

Given a stretching parameter, L, we use the standard differentiation matrices to find the derivative of $\boldsymbol{u}$ in terms of Chebyshev polynomials [7] and then use the above transformations to stretch the result to the real line.

The nature of the RCP means that they are well suited to both Dirichlet and Neumann boundary conditions [5]. In the numerical simulations given here nothing extra, beyond using the RCP, was done to take the boundary conditions into consideration.

\section{$3 \quad$ Exponential integrators}

Many optical propagation problems are described by the general PDE

$$
\frac{\partial u}{\partial t}=\mathcal{L} u+\mathcal{N}(u)
$$

where $\mathcal{L}$ is a linear differential operator (usually of second order) and $\mathcal{N}(\mathfrak{u})$ is an autonomous (although not necessarily) nonlinear operator. 
The use of the Chebyshev polynomials allows the discretization of the linear operator $\mathcal{L}$ and turned the PDE into a system ordinary differential equations (ODE). However, the discretised $\mathcal{L}$ is dense and the resulting ODE system is stiff. The higher order accuracy of the spectral method reduces the amount of computation, despite the resulting dense matrix, as fewer points will be needed to perform the computation to achieve a required accuracy. The stiffness of the system is a more significant computational issue and would normally require small steps in the propagation dimension or an implicit time-stepping scheme in order to maintain accuracy. This counteracts the benefits of the spectral scheme.

Here, we take advantage of the so-called exponential integrators (Minchev and Wright [8] reviewed the subject), which have gained renewed interest in recent years. The basic idea is to use the function $\exp (-\mathcal{L} t)$ as an integrating factor for the PDE. Then one integrates from 0 to $\Delta t$ where $\Delta t$ is the forward time-step size. The resulting expression is formally exact and the role of different exponential integration schemes is to approximate the integral in the propagation rule

$$
u_{j+1}=e^{\Delta t \mathcal{L}} u_{j}+e^{\Delta t \mathcal{L}} \int_{0}^{\Delta t} e^{-\tau \mathcal{L}} N\left(u_{j}\left(t_{j}+\tau\right)\right) d \tau,
$$

where the subscript $j$ indicates the $j$ th step. We employed the schemes developed by Krogstad [9] and Kassam \& Trefethen [10]. Both schemes resemble the classic Runge-Kutta schemes for solving non-stiff ODEs but rely on the efficient computation of the so-called $\phi$-functions

$$
\phi_{0}(z)=e^{z}, \quad \phi_{j+1}(z)=\frac{\phi_{j}(z)-1 / j !}{z},
$$

where the argument of $\phi_{j}$ is, in the case of the RCP, a dense matrix. The calculation of $\phi_{j}$ is only required once at the start of the simulation.

Calculating a given $\phi_{j}$ is computationally difficult due to the need to find the matrix exponential and the possibility of a catastrophic cancellation [11]. 
Cox and Matthews [11] suggest using a Taylor series expansion for $\phi_{0}(z)$ when $z$ is small but there is a range of values where neither the Taylor series nor the exact expression for $\phi_{0}(z)$ are numerically accurate enough. Kassam and Trefethen [10] suggest a complex contour integral technique to calculate $\phi_{0}(z)$ which is successful but the contour of the integral is arbitrary and may need some trial and error to find. Here the squaring and scaling technique has been used for matrix exponentials and high order Padé approximations have been used for the $\phi_{j}(z)[12]$.

\section{Results}

For the purposes of testing we solve numerically the $(1+1)$-dimensional NLS, $(1+1)$-dimensional and $(2+1)$-dimensional CQNLS. The $(2+1)$-dimensional CQNLS is

$$
i \frac{\partial u}{\partial t}+\frac{\partial^{2} u}{\partial x^{2}}+\frac{\partial^{2} u}{\partial y^{2}}+\left(|u|^{2}-|u|^{4}\right) u=0,
$$

and if the quintic nonlinearity and one of the transverse derivatives are dropped we have the NLS. The benefits of using these PDEs, apart from the fact they are commonly used to model optical propagation, is that the one dimensional versions have well known soliton solutions and all have conserved qualities which are used to monitor the scheme's accuracy.

The scheme successfully handled propagating both bright and dark soliton solutions in the one dimensional NLS and CQNLS. Bright and dark solitons require Dirichlet and Neumann boundary conditions respectively. Using the well known breather soliton of the NLS [13, pg. 69], which has the simple form of $u=2 \operatorname{sech} y$ at $t=0$, we compared the RCP method with the split-step Fourier method. Figure 1 shows the beam profiles according to each method at $t=20$. The RCP method is indistinguishable from the exact solution while the Fourier method has shed energy as radiation and the beam is beginning to move to the left. For both methods the number of transverse 


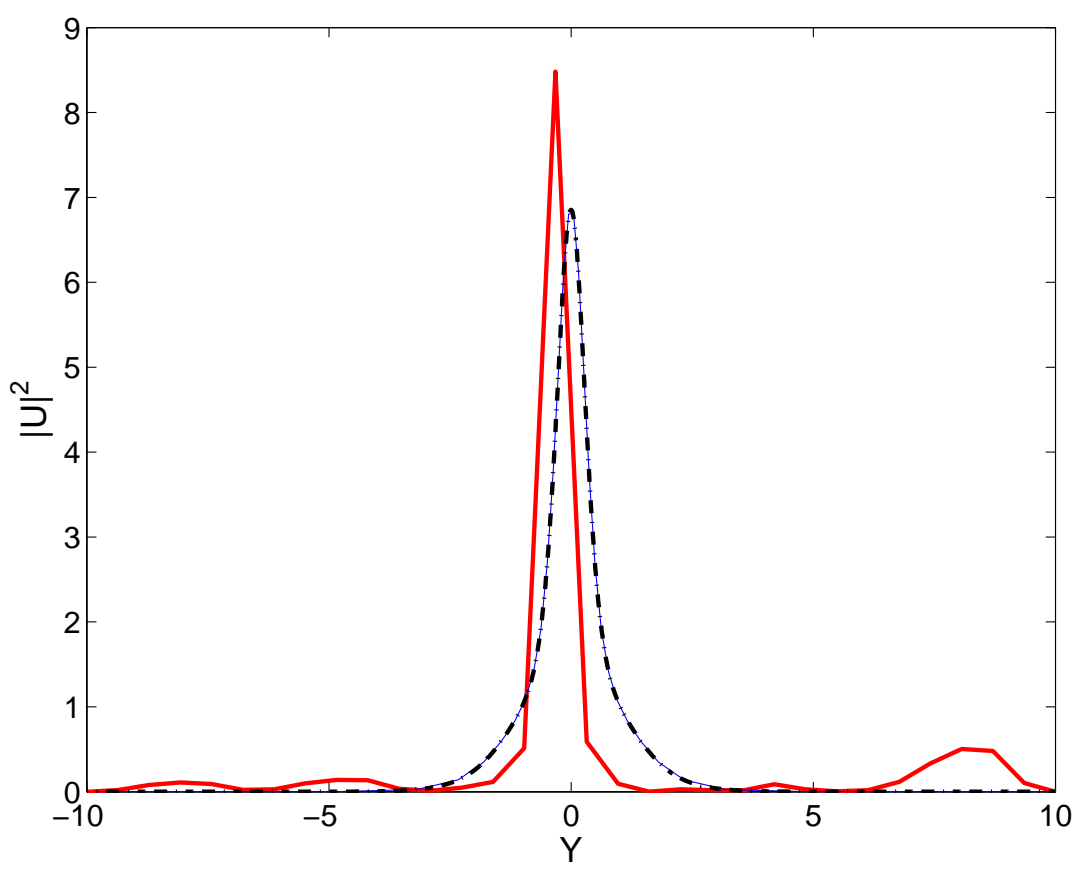

Figure 1: The RCP method (thin line) and the standard split-step Fourier method (thick line) are compared to the exact breather soliton (dashed line) of the NLS at $t=20$. Both methods use 32 points in the transverse dimension (width 20 units) and have a time-step of 0.01. At this scale the RCP method is indistinguishable from the exact solution. 


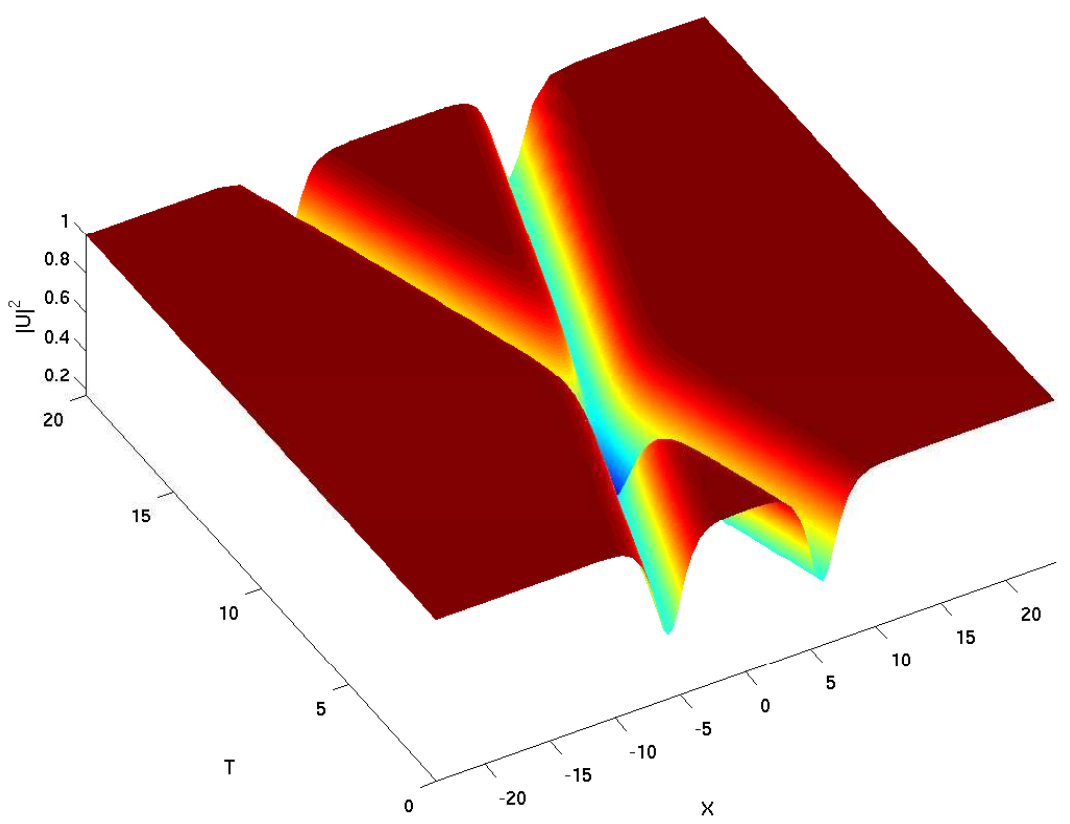

Figure 2: The collision of two dark solitons of the nonlinear Schrödinger equation.

points is 32 and the time-step is 0.01 . Using the same initial condition we integrated the NLS with the RCP method until $t=100$. Again, the profile is indistinguishable from the exact solution. For the Fourier method to produce a beam profile replicating the exact solution it requires 512 points in the transverse dimension and at least an order of magnitude smaller time-step. To avoid the possibility of the onset of high frequency instability inherent to the split step method [3] the time-step had to be reduced to $4 \times 10^{-4}$.

Figure 2 shows two dark solitons colliding, interacting nonlinearly, and then passing through each other as if unaffected. The initial profile used for 


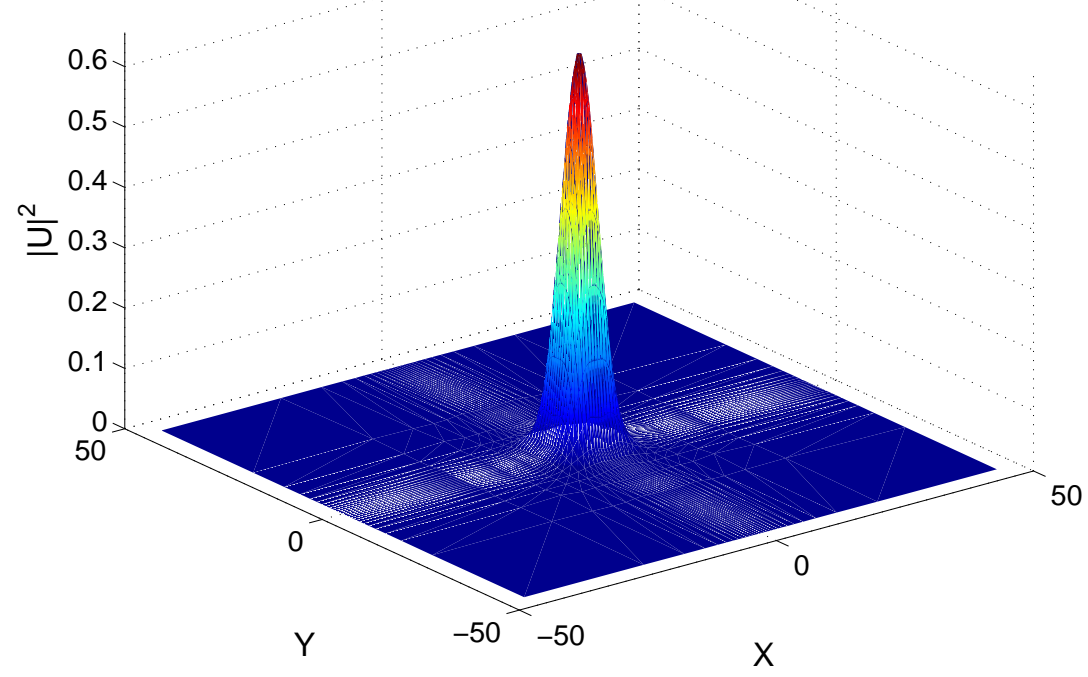

Figure 3: The beam intensity of an initial Gaussian beam propagated according to the $(2+1)$-dimensional cubic-quintic nonlinear Schrödinger equation after 100 time units.

this simulation was given by an exact solution of the NLS

$$
u(y, t)=\frac{\left(a_{3}-a_{1}\right) \cosh (\mu t)-\sqrt{a_{1} a_{3}} \cosh (2 p y)+i \mu \sinh (\mu t)}{\sqrt{a_{3}} \cosh (\mu t)+\sqrt{a_{1}} \cosh (2 p y)} e^{i a_{3} t},
$$

where $\mu=2 \sqrt{a_{1}\left(a_{3}-a_{1}\right)}$ and $p=\sqrt{a_{3}-a_{1}}$ [13, p. 99]. For the simulation we used $a_{1}=0.5$ and $a_{3}=1$ while we set $t=-8$ in the expression above so the collision occurs during the simulation. The numerical scheme successfully reproduced the dynamical behaviour of the exact solution.

The $(2+1)$-dimensional CQNLS is not integrable so exact soliton solutions are not known although good approximations are made using variational calculus [13]. The non-integrable nature of the equation means that energy 
of the initial pulse will be shed as radiation as the pulse reshapes into the soliton solution of the CQNLS. The scheme successfully handles the radiation. Figure 3 shows the result of propagating a Gaussian initial beam for 100 time steps during which time it reshapes.

As an application outside the field of optics we considered the FisherKolmogorov equation [14]

$$
\frac{\partial u}{\partial t}=\frac{\partial^{2} u}{\partial x^{2}}+u-u^{2}, \quad u(-\infty, t)=1, \quad u(\infty, t)=0,
$$

which has non-homogeneous boundary conditions on the real line. The Fisher-Kolmogorov equation has the same generic form that was presented in Section 3.

The equation has travelling wave solutions and the theory states that almost any initial condition which satisfies the boundary condition will reshape into a traveling wave solution with wave speed 2. Figure 4 presents several 'snapshots' of the traveling wave moving to the right.

The scheme can be improved by developing a method to optimally or adaptively choose $\mathrm{L}$ the stretching parameter of the RCP. If $\mathrm{L}$ could be chosen adaptively then the density of the discrete points in the transverse dimension could be changed as a beam moved so that more points were available to describe the beam profile where it is changing most. Further, computing the $\phi$-functions is essential for the exponential integrators so any work that improves the calculation of $\phi_{j}$ improves the scheme overall.

\section{Conclusion}

We have demonstrated the use of coupling RCP with exponential integrators to study beam propagation in optical situation. The method avoids the 


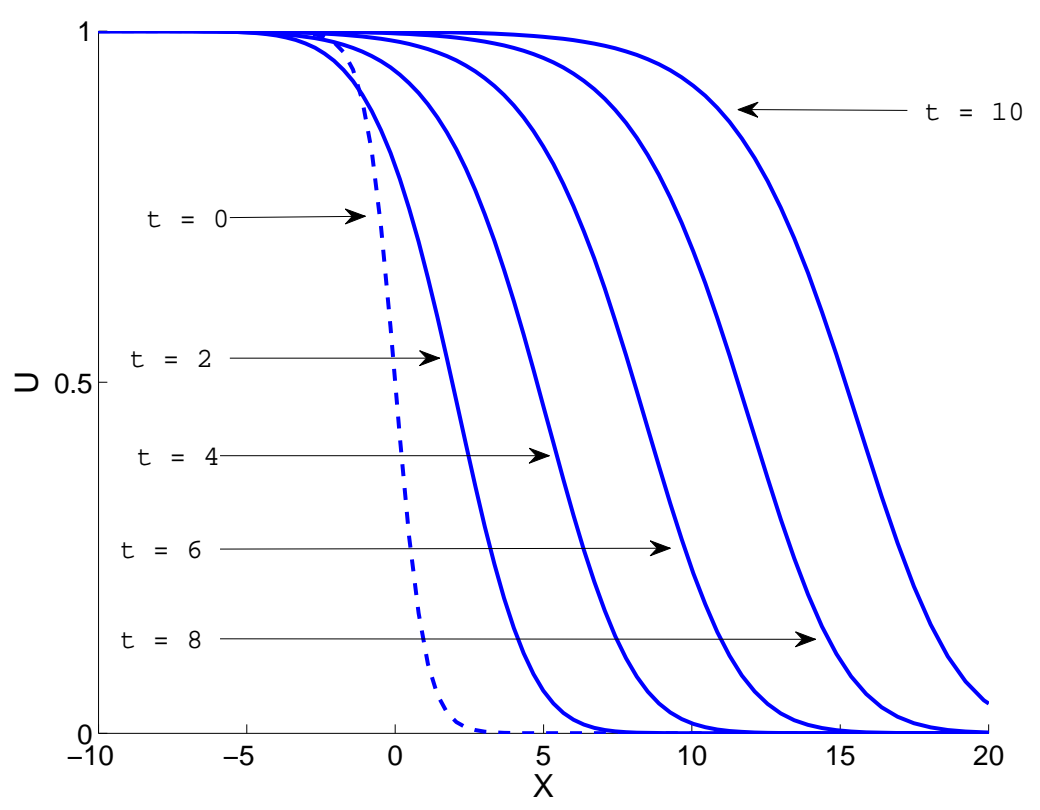

Figure 4: The solution of the Fisher-Kolmogorov equation with a tanh initial condition (dashed line) quickly resolves into a traveling wave with speed 2 . 
common deficiencies that periodic boundary conditions impose while maintaining the accuracy and speed of a spectral scheme. We have seen gains of two orders of magnitude in the time step size and an order of magnitude reduction in the number of points required in the transverse dimension when compared to the more commonly used split-step Fourier methods.

\section{References}

[1] L. N. Trefethen. Spectral Methods in Matlab. Siam, Philadelphia, PA, 2000.

http://www.comlab.ox.ac.uk/nick.trefethen/spectral.html C62

[2] M. Frigo and S. G. Johnson. The Design and Implementation of FFTW3. Proceedings of the IEEE, 93, 2005, 216-231.

doi:10.1109/JPROC.2004.840301; Fastest Fourier Transform in the West. http://www.fftw.org/ C62

[3] J. A. C. Weideman and B. M. Herbst. Split-step methods for the solution of the nonlinear Schrödinger equation. SIAM J. Numer. Anal., 23, 1986, 485-507. http://www. jstor.org/stable/2157521 C62, C68

[4] A. Taflove and S. C. Hageness. Computational Electrodynamics: The Finite-Difference Time-Domain Method.Norwood, MA: Artech, 2000. $\mathrm{C} 62$

[5] J. P. Boyd. Chebyshev and Fourier Spectral Methods. Dover Publications, 2nd edition, 2000. C63, C64

[6] J. P. Boyd. Spectral methods using rational basis functions on an infinite interval. J. Comput. Phys., 69, 1987, 112-142. doi:10.1016/0021-9991(87)90158-6 C63 
[7] J. A. C. Weideman and S. C. Reddy. A Matlab differentiation matrix suite. ACM Transactions on Mathematical Software, 26, 2000, 465-519. doi:10.1145/365723.365727 C64

[8] B. Minchev and W. Wright. A review of exponential integrators for first order semi-linear problems. Preprint Numerics No. 2/2005. Norwegian University of Science and Technology. http://www.math.ntnu.no/preprint/numerics/2005/N5-2005.pdf C65

[9] S. Krogstad. Generalized integrating factor methods for stiff pdes. J. Comput. Phys., 203, 2002, 72-88. doi:10.1016/j.jcp.2004.08.006 C65

[10] A. Kassam and L. N. Trefethen. Fourth-order time-stepping for stiff pdes. SIAM J. Sci. Comput., 6, 2005, 1214-1244. doi:10.1137/S1064827502410633 C65, C66

[11] S. M. Cox and P. C. Matthews. Exponential time differencing for stiff systems. J. Comput. Phys., 176, 2002, 430-455. doi:10.1006/jcph.2002.6995 C65, C66

[12] H. Berland, B. Skaflestad and W. M. Wright.Expint - A Matlab package for exponential integrators. ACM Trans. Math. Softw.,33, 2007, 4. doi:10.1145/1206040.1206044 C66

[13] N. N. Akhmediev and A. Ankiewicz. Solitons - Nonlinear pulses and beams. Chapman \& Hall, 1997. C66, C69

[14] R. A. Fisher. The wave of advance of advantageous genes. Ann. Eugenics, 7, 1937, 353-369. http:

//digital.library.adelaide.edu.au/dspace/handle/2440/15125 C70 


\section{Author addresses}

1. I. N. Towers, School of Physical, Environmental \& Mathematical Sciences, University of New South Wales, Canberra, Australia. mailto: i. towers@adfa.edu.au

2. Z. Jovanoski, School of Physical, Environmental \& Mathematical Sciences, University of New South Wales, Canberra, Australia. 